\title{
AVALIAÇÃO DOS NÍVEIS DE ANSIEDADE DE UMA AMOSTRA DE ESCOLARES NO RIO DE JANEIRO ATRAVÉS DA Escala Multidimensional de Ansiedade PARA CRIANÇAS (MASC-VB)
}

Transtornos de ansiedade representam uma das mais comuns e debilitantes formas de psicopatologia na infância e adolescência. O objetivo deste trabalho foi avaliar as propriedades psicométricas da Escala Multidimensional de Ansiedade para Crianças (MASC-VB) e medir os níveis de ansiedade em crianças e adolescentes no Rio de Janeiro. Os dados obtidos reforçam o bom desempenho psicométrico da escala para uso na população brasileira e apontam para um alarmante achado com relação aos altos níveis de ansiedade em crianças amostradas na Rocinha.

\section{BANCA:}

Jesus Landeira Fernandez (Orientadora)

Bernard Pimentel Rangé

Helenice Charchat Fichman

Data da defesa: 17/03/2009 